\title{
EXPONENTIAL BOUNDS OF RUIN PROBABILITIES FOR NON-HOMOGENEOUS RISK MODELS
}

BY

\author{
QIANQIAN ZHOU* (NANJING), ALEXANDER SAKHANENKO** (Novosibirsk), \\ AND JUNYI GUO O $^{* * *}$ (TIANJIN)
}

\begin{abstract}
Lundberg-type inequalities for ruin probabilities of nonhomogeneous risk models are presented. By employing the martingale method, upper bounds of ruin probabilities are obtained for general risk models under weak assumptions. In addition, several risk models, including the newly defined united risk model and a quasi-periodic risk model with interest rate, are studied.
\end{abstract}

2020 Mathematics Subject Classification: Primary 91G05; Secondary $60 \mathrm{~K} 10$.

Key words and phrases: non-homogeneous risk model, martingale method, ruin probability, Lundberg-type inequality.

\section{INTRODUCTION}

Research on the ultimate ruin probability $\psi(u)$, i.e., the probability that the reserves ever drop below zero:

$$
\psi(u):=\mathbf{P}\left[\inf _{t \geqslant 0} R(t)<0 \mid R(0)=u\right],
$$

where $R(t)$ is a risk reserve process with initial reserve $R(0)=u>0$, has attracted increasing attention since classic works of Lundberg [20] and Cramér [9], [10]. A substantial amount of work has been devoted to finding the ruin probabilities and their upper bounds: see, e.g., Dickson [11], Gerber [12], and Rolski et al. [22]. However, the most outstanding result about the behavior of $\psi(u)$ is the

* Supported by NUPTSF (No. NY221026).

** Supported by the program of fundamental scientific research of the SB RAS № I.1.3., project № 0314-2019-0008.

*** Supported by NSFC (No. 11931018) and Tianjin NSF (19JCYBJC30400). 
Lundberg inequality, which states that under appropriate assumptions (see [2] and [13] for more details) there exists $L$ such that

$$
\psi(u) \leqslant e^{-L u} \quad \text { for all } u \geqslant 0 .
$$

The largest number $L$ is called the adjustment coefficient or Lundberg exponent. Further, the following Lundberg-type inequality has also been studied:

$$
\forall u \geqslant 0, \quad \psi(u) \leqslant C e^{-L u} \quad \text { with } C<\infty \text { and } L>0 .
$$

In classical works on homogeneous risk models, both inter-occurrence times and claim sizes are assumed to be i.i.d. random variables. However, in reality, both factors are influenced by the economic environment. For instance, inflation and interest rate can affect the evolution of the reserves of the company. Thus the assumptions of homogeneity of inter-occurrence times and claim amounts can be too restrictive for practical use, and non-homogeneous models better reflect the real insurance activities.

Recently, the interest in non-homogeneous risk models has considerably increased. In such models the inter-occurrence times or/and the claim sizes are independent but not necessarily identically distributed. Blaževičius et al. [7], Castañer et al. [8], Lefèvre and Picard [19], and Vernic [25] investigated non-homogeneous risk models with independent, identically distributed inter-occurrence times but not necessarily identically distributed claims. For more details about non-homogeneous risk models with independent and identically distributed claim sizes, and independent but not identically distributed inter-occurrence times one can refer to Bernackaitè and Šiaulys [6], Ignatov and Kaishev [15] and Tuncel and Tank [24].

However, we have found only a few works in which estimates of the types (1.1) or (1.2) are obtained. In Andrulytè et al. [1], Kievinaitè and Šiaulys [16], and Kizinevič and Šiaulys [18], a non-homogeneous renewal risk model is considered where the claim sizes and inter-occurrence times are both independent but not necessarily identically distributed, and the Lundberg-type inequality is investigated.

This paper considers a non-homogeneous compound Poisson risk model and studies its ruin probability $\psi(u)$. Our purpose is to obtain an exponential upper bound of the ultimate ruin probability under general assumptions.

The paper is organized as follows. In Section 2, we present our main results. First, we study the non-homogeneous compound Poisson risk model without interest rate. By employing the martingale method, the Lundberg inequality for this model is obtained. Second, a non-homogeneous risk model with interest rate is studied and the Lundberg inequality is also obtained. In Section 3, applications of our main results are considered, with a special focus on new behaviors of the probability $\psi(u)$. First, periodic and quasi-periodic risk models with interest rate, which are also automatically non-homogeneous, are investigated. Second, a new non-homogeneous risk model, which is called the united risk model, is defined, and the corresponding Lundberg inequality is obtained. In the last section, we compare 
our results with the previous results of Andrulytė et al. [1], Kievinaitè and Šiaulys [16], and Kizinevič and Šiaulys [18].

\section{MAIN RESULTS}

2.1. Non-homogeneous risk model without interest rate. We begin by considering a general risk model in which the distribution of each inter-arrival time $\theta_{n}:=$ $T_{n}-T_{n-1}$ may depend on $T_{n-1}$.

Model A: Let $N(t)$ be a non-homogeneous Poisson process with non-decreasing accumulated intensity function $\Lambda(t):=\mathbf{E} N(t)<\infty$ where $N(0)=\Lambda(0)=0$. Denote by $Z(t) \geqslant 0$ the claim size in case the claim arrives at time $t>0$, and for each $j=0,1,2, \ldots$, let

$$
T_{j}:=\min \{t \geqslant 0: N(t) \geqslant j\} .
$$

Then it is natural to introduce the risk reserve process $R(t)$ by

$$
R(t)=u+p(t)-\sum_{j=1}^{N(t)} Z\left(T_{j}\right), \quad \forall t \geqslant 0,
$$

where $u=R(0)>0$ is the initial reserve, $p(t) \geqslant 0$ is the aggregate premium income over $[0, t]$, which is assumed to be non-decreasing, and $\left\{Z\left(T_{j}\right)\right\}_{j \geqslant 1}$ are the claim sizes which are supposed to be independent but not identically distributed.

It is easy to see that model $\mathrm{A}$ is more general than the classical compound Poisson risk model which supposes that $N(t)$ is a homogeneous Poisson process, $\left\{Z\left(T_{j}\right)\right\}_{j \geqslant 1}$ are i.i.d. random variables and the premium rate is a constant.

For mathematical convenience, we introduce the claim surplus process

$$
S(t)=\sum_{j=1}^{N(t)} Z\left(T_{j}\right)-p(t)
$$

thus $R(t)=u-S(t)$. Then the ultimate ruin probability can be expressed as

$$
\psi(u)=\mathbf{P}\left(\sup _{t \geqslant 0} S(t)>u\right) .
$$

We suppose that the random processes $N(\cdot)$ and $Z(\cdot)$ are independent. From Kingman [17] and Paulsen [21] we can see that for any real number $h \geqslant 0$ and any $t>0$, if $Q(h, t)=\mathbf{E} e^{h Z(t)}-1$ exists and is continuous in $t \in(0, \infty)$, then the claim surplus process $S(t)$ defined in (2.1) satisfies

$$
\mathbf{E} e^{h S(t)}=\exp \left\{\int_{0}^{t} Q(h, x) d \Lambda(x)-h p(t)\right\}, \quad \forall h \geqslant 0, t>0,
$$


where $\Lambda(t)=\mathbf{E} N(t)$ is the accumulated intensity function of $N(t)$ and $p(t)$ is the aggregate premium income over $[0, t]$. Note that the formula in $[2.2)$ is valid for a larger class of non-homogeneous processes $S(t)$ with independent increments.

Before studying model A, we first state the following basic theorem.

THEOREM 2.1. Let $W(t)$ be a separable process with independent increments. Then for any real numbers $x$ and $T, h \geqslant 0$,

$$
\mathbf{P}\left(\sup _{0 \leqslant t \leqslant T} W(t)>x\right) \leqslant e^{-h x} \sup _{0 \leqslant t \leqslant T} \mathbf{E} e^{h W(t)} .
$$

Consequently, for all $x$ and $h \geqslant 0$,

$$
\mathbf{P}\left(\sup _{t \geqslant 0} W(t)>x\right) \leqslant e^{-h x} \sup _{t \geqslant 0} \mathbf{E} e^{h W(t)} .
$$

Before proving Theorem 2.1 we first introduce a key lemma which comes from [26].

LEMMA 2.1. If random variables $Y_{1}, Y_{2}, \ldots$ are mutually independent, then for any $n \geqslant 1$ and any real number $x$ and $h \geqslant 0$,

$$
\mathbf{P}\left(\sup _{1 \leqslant k \leqslant n} W_{k}>x\right) \leqslant e^{-h x} \sup _{1 \leqslant k \leqslant n} \mathbf{E} e^{h W_{k}},
$$

where $W_{k}:=Y_{1}+\cdots+Y_{k}$. Consequently, for any real number $x$ and $h \geqslant 0$,

$$
\mathbf{P}\left(\sup _{k \geqslant 1} W_{k}>x\right) \leqslant e^{-h x} \sup _{k \geqslant 1} \mathbf{E} e^{h W_{k}} .
$$

Proof of Theorem 2.1 Since $W(t)$ is a separable process, there exists a sequence $t_{1}, t_{2}, \ldots \in[0, T]$ such that

$$
\max _{1 \leqslant k \leqslant n} W\left(t_{k}\right) \uparrow \sup _{k \geqslant 1} W\left(t_{k}\right)=\sup _{0 \leqslant t \leqslant T} W(t) .
$$

Thus

$$
\mathbf{P}\left(\sup _{0 \leqslant t \leqslant T} W(t)>x\right)=\lim _{n \rightarrow \infty} \mathbf{P}\left(\max _{1 \leqslant k \leqslant n} W\left(t_{k}\right)>x\right) .
$$

Let $\left\{t_{1}, \ldots, t_{n}\right\}=\left\{t_{1}^{*}<\cdots<t_{n}^{*}\right\}$. Then

$$
\max _{1 \leqslant k \leqslant n} W\left(t_{k}\right)=\max _{1 \leqslant k \leqslant n} \sum_{j=1}^{k}\left[W\left(t_{j}^{*}\right)-W\left(t_{j-1}^{*}\right)\right],
$$

where $t_{0}^{*}=0, W\left(t_{0}^{*}\right)=0$. Since $\left\{W\left(t_{j}^{*}\right)-W\left(t_{j-1}^{*}\right)\right\}_{j \geqslant 1}$ are independent, from Lemma 2.1 we have

$$
\mathbf{P}\left(\max _{1 \leqslant k \leqslant n} W\left(t_{k}\right)>x\right) \leqslant e^{-h x} \max _{1 \leqslant k \leqslant n} \mathbf{E} e^{h W\left(t_{k}\right)} .
$$

Letting $n \rightarrow \infty$ yields 2.3); and (2.4) follows by letting $T \rightarrow \infty$. 
Note that Theorem 2.1 allows us to obtain the following results on the ruin probabilities for model A.

THEOREM 2.2. Suppose the claim surplus process $S(t)$ defined in (2.1) is a separable process with independent increments. Then for any $u>0$ and $T, h \geqslant 0$,

$$
\psi(u, T):=\mathbf{P}\left(\sup _{0 \leqslant t \leqslant T} S(t)>u\right) \leqslant e^{-h u} \sup _{0 \leqslant t \leqslant T} \mathbf{E} e^{h S(t)} .
$$

Consequently, for any $u>0$ and $h \geqslant 0$,

$$
\psi(u)=\mathbf{P}\left(\sup _{t \geqslant 0} S(t)>u\right) \leqslant e^{-h u} \sup _{t \geqslant 0} \mathbf{E} e^{h S(t)} .
$$

Theorem 2.2 and 2.2) immediately imply the following Lundberg inequality for the ruin probability in model $\mathrm{A}$.

COROLLARY 2.1. Under the conditions of Theorem 2.2. if $S(t)$ satisfies (2.2) then the Lundberg inequality (1.1) holds with $L=\underline{L}$, where

$$
\underline{L}=\sup \left\{h \geqslant 0: \sup _{t \geqslant 0}\left[\int_{0}^{t} Q(h, x) d \Lambda(x)-h p(t)\right] \leqslant 0\right\} .
$$

The homogeneous compound Poisson risk model is a special case of model A with

$$
\mathbf{E} e^{h S(t)}=\exp \left\{t\left[\lambda\left(\mathbf{E} e^{h Z}-1\right)-h p\right]\right\}, \quad \forall t>0, h \geqslant 0 .
$$

Then Corollary 2.1 implies the classical Lundberg inequality (1.1) with

$$
L=\sup \left\{h \geqslant 0: \lambda\left(\mathbf{E} e^{h Z}-1\right) \leqslant h p\right\} .
$$

REMARK 2.1. From 2.6 it can be seen that the Lundberg inequality obtained by our method is better than its classical form, which can be found in [2], [13], [22], because we do not exclude the cases when $\mathbf{E} e^{L S(t)}<1$ and/or $\mathbf{E} S(t)=-\infty$.

2.2. Non-homogeneous risk model with interest rate. It is well-known that all risk models under interest rates are automatically non-homogeneous. This gives us additional motivation in our research of non-homogeneous risk models. In this subsection we consider a non-homogeneous compound Poisson risk model with interest rate.

Model B: Let $N(t), Z(t)$ and $p(t)$ be as in model A, and suppose that for any $t_{2}>t_{1} \geqslant 0$ the risk reserve process $R(t)$ has the following property:

$$
R\left(t_{2}\right)=\left(1+\alpha\left(t_{1}, t_{2}\right)\right) R\left(t_{1}\right)+\left(1+\beta\left(t_{1}, t_{2}\right)\right)\left[p\left(t_{2}\right)-p\left(t_{1}\right)\right]-\left[q\left(t_{2}\right)-q\left(t_{1}\right)\right],
$$


where $\left(1+\alpha\left(t_{1}, t_{2}\right)\right) R\left(t_{1}\right)$ is the accumulated value of $R\left(t_{1}\right)$ from $t_{1}$ to $t_{2}$ under interest rate $\alpha\left(t_{1}, t_{2}\right)$ and $\left(1+\beta\left(t_{1}, t_{2}\right)\right)\left[p\left(t_{2}\right)-p\left(t_{1}\right)\right]$ is the accumulated value of premiums collected from $t_{1}$ to $t_{2}$ under interest rate $\beta\left(t_{1}, t_{2}\right)$.

Here $q(t)$ denotes the total claims over the time interval $[0, t]$. Of course, we will first consider the case when $q(t)=\sum_{j=1}^{N(t)} Z\left(T_{j}\right)$. We suppose that

$$
\beta\left(t_{1}, t_{2}\right) \geqslant 0 \quad \text { and } \quad \alpha\left(t_{1}, t_{2}\right) \geqslant e^{r\left(t_{2}\right)-r\left(t_{1}\right)}, \quad \forall t_{2}>t_{1} \geqslant 0,
$$

where $r(t)$ is non-decreasing and $r(0)=0$. From 2.7) and (2.8) we have

$$
R\left(t_{2}\right) \geqslant e^{r\left(t_{2}\right)-r\left(t_{1}\right)} R\left(t_{1}\right)-\left[S\left(t_{2}\right)-S\left(t_{1}\right)\right]
$$

with $S(t)=q(t)-p(t)$. Hence for all $t_{2}>t_{1} \geqslant 0$,

$$
e^{-r\left(t_{2}\right)} R\left(t_{2}\right) \geqslant e^{-r\left(t_{1}\right)} R\left(t_{1}\right)-e^{-r\left(t_{2}\right)}\left[S\left(t_{2}\right)-S\left(t_{1}\right)\right] .
$$

From (2.9) for any $0=t_{0}<t_{1}<\cdots<t_{m}=t$ we have

$$
\begin{aligned}
e^{-r(t)} R(t)-u & =\sum_{k=1}^{m}\left[e^{-r\left(t_{k}\right)} R\left(t_{k}\right)-e^{-r\left(t_{k-1}\right)} R\left(t_{k-1}\right)\right] \\
& \geqslant-\sum_{k=1}^{m} e^{-r\left(t_{k}\right)}\left[S\left(t_{k}\right)-S\left(t_{k-1}\right)\right] .
\end{aligned}
$$

If $S(t)$ is a function of bounded variation, then standard arguments allow us, as $m \rightarrow \infty$, to replace the sum on the right hand side of 2.10 by an integral:

$$
e^{-r(t)} R(t) \geqslant u-\int_{0}^{t} e^{-r(x)} d S(x)=: u-Y(t), \quad \forall t>0 .
$$

In addition, from (2.11) we have

$$
\begin{aligned}
\psi(u) & =\mathbf{P}\left(\inf _{t \geqslant 0} R(t)<0\right)=\mathbf{P}\left(\inf _{t \geqslant 0} e^{-r(t)} R(t)<0\right) \\
& \leqslant \mathbf{P}\left(\sup _{t \geqslant 0} Y(t)>u\right) .
\end{aligned}
$$

It is easy to see that if the integral in 2.11) makes sense for some process $S(t)$ with independent increments and non-random $r(t)$, then $Y(t)$ is also a process with independent increments. This fact and (2.11) imply the following assertion.

THEOREM 2.3. Suppose that 2.11) holds where $Y(t)$ is a separable process with independent increments. Then all the assertions of Theorem 2.2 hold with $Y(t)$ in place of $S(t)$. 
COROLLARY 2.2. Under the assumptions of Theorem 2.3. assume also that

$$
\mathbf{E} e^{h Y(t)}=e^{a(h, t)}
$$

where

$$
a(h, t):=-h \int_{0}^{t} e^{-r(x)} d p(x)+\int_{0}^{t} Q\left(h e^{-r(x)}, x\right) d \Lambda(x) .
$$

Then the Lundberg inequality (1.1) holds with $L=L^{*}$, where

$$
L^{*}=\sup \left\{h \geqslant 0: \sup _{t \geqslant 0} a(h, t) \leqslant 0\right\} \text {. }
$$

This is an analogue of Corollary 2.1 for models without interest rates.

REMARK 2.2. Suppose the representation (2.2) holds with a non-decreasing function $\Lambda(\cdot)$ and natural continuity assumptions on $Q(\cdot, \cdot)$ in the domain where it is finite. It is not difficult to see that if the integral in 2.11 makes sense for some process $S(t)$ with independent increments and a non-random function $r(t)$ of bounded variation then $Y(t)$ is also a process with independent increments such that

$\mathbf{E} e^{h Y(t)}=e^{a(h, t)} \quad$ with $\quad a(h, t):=-h \int_{0}^{t} e^{-r(x)} d p(x)+\int_{0}^{t} Q\left(h e^{-r(x)}, x\right) d \Lambda(x)$.

We refer to Paulsen [21] for more details. Thus the assumption (2.13) in Corollary 2.2 is reasonable.

REMARK 2.3. Note that Theorems 2.2 and 2.3 immediately follow from Theorem 2.1.

\section{APPLICATIONS OF MAIN RESULTS}

In this section, we consider several applications of our general results with a special focus on the behavior of the probability $\psi(u)$ in non-homogeneous cases. First, we investigate periodic and quasi-periodic risk models which are automatically nonhomogeneous. Second, we put forward a new non-homogeneous risk model and study its probability of ruin.

3.1. Periodic and quasi-periodic risk models. In this subsection, we study risk models in a periodic environment. For the classical periodic case, Asmussen and Rolski [3] have shown that the adjustment coefficient in the periodic risk model is the same as for the standard time-homogeneous compound Poisson risk process obtained by averaging the parameters over one period. Here we study the general periodic risk models with interest rates. 
In the following, under the assumptions of Theorem 2.3, we also assume that $\Lambda(t)$ has density $\Lambda^{\prime}(t)$ and the aggregate premium income $p(t)$ has density $p^{\prime}(t)$. Then 2.13) can be rewritten as

$$
\mathbf{E} e^{h Y(t)}=\exp \left\{-\int_{0}^{t} h e^{-r(x)} p^{\prime}(x) d x+\int_{0}^{t}\left(\mathbf{E} e^{h e^{-r(x)} Z(x)}-1\right) \Lambda^{\prime}(x) d x\right\}
$$

for all $t>0$.

COROLLARY 3.1. Suppose there exist real numbers $l, t_{0}, \widetilde{L}>0$ such that for any $t \geqslant t_{0}$,

$$
\mathbf{E} e^{\widetilde{L}(Y(t+l)-Y(t))} \leqslant 1 .
$$

Then under the assumptions of Theorem 2.3. the assertions of the theorem hold for each $h \in[0, \widetilde{L}]$ with

$$
\sup _{t \geqslant 0} \mathbf{E} e^{h Y(t)}=\sup _{0 \leqslant t<t_{0}+l} \mathbf{E} e^{h Y(t)}
$$

In addition, for any $u>0$,

$$
\psi(u) \leqslant \inf _{h \in[0, \widetilde{L}]}\left\{e^{-h u} \sup _{0 \leqslant t<t_{0}+l} \mathbf{E} e^{h Y(t)}\right\} \leqslant C_{1} e^{-\widetilde{L} u},
$$

where $C_{1}:=\sup _{0 \leqslant t<t_{0}+l} \mathbf{E} e^{\widetilde{L} Y(t)}$.

Proof. For any $t \geqslant t_{0}+l$, the random variables $\Delta_{t, l}:=Y(t)-Y(t-l)$ and $Y(t-l)$ are independent. Hence for each $h \in[0, \widetilde{L}]$,

$$
\begin{aligned}
\mathbf{E} e^{h \Delta_{t, l}} & \leqslant\left(\mathbf{E} e^{\widetilde{L} \Delta_{t, l}}\right)^{h / \widetilde{L}} \leqslant 1 \\
\mathbf{E} e^{h Y(t)} & =\mathbf{E} e^{h \Delta_{t, l}} \mathbf{E} e^{h Y(t-l)} \leqslant \mathbf{E} e^{h Y(t-l)} .
\end{aligned}
$$

For any $t \geqslant t_{0}+l$, by the same procedure as in deriving (3.3) we have

$$
\mathbf{E} e^{h Y(t-l)} \leqslant \mathbf{E} e^{h Y(t-2 l)}
$$

if $t-l \geqslant t_{0}+l$, and otherwise $\mathbf{E} e^{h Y(t)} \leqslant \sup _{t<t_{0}+l} \mathbf{E} e^{h Y(t)}$.

It is not difficult to see that for any $t \geqslant t_{0}+l$ and $h \in[0, \widetilde{L}]$ we can repeat the above procedure $i$ times until $t-i l<t_{0}+l$ with

$$
\mathbf{E} e^{h Y(t)} \leqslant \sup _{t<t_{0}+l} \mathbf{E} e^{h Y(t)} .
$$

Hence

$$
\sup _{0 \leqslant t<t_{0}+l} \mathbf{E} e^{h Y(t)} \leqslant \sup _{t \geqslant 0} \mathbf{E} e^{h Y(t)} \leqslant \sup _{0 \leqslant t<t_{0}+l} \mathbf{E} e^{h Y(t)}, \quad \forall h \in[0, \widetilde{L}],
$$

i.e.,

$$
\sup _{t \geqslant 0} \mathbf{E} e^{h Y(t)}=\sup _{0 \leqslant t<t_{0}+l} \mathbf{E} e^{h Y(t)}, \quad \forall h \in[0, \widetilde{L}] .
$$

The other assertions come from Theorem 2.3 and Corollary 2.2 . 
THEOREM 3.1. Suppose there exists a real number $l>0$ such that for each $h \in[0, L(Y(l))]$ and any $t \geqslant 0$,

$$
e^{r(t+l)}\left[\mathbf{E} e^{h e^{-r(t+l)} Z(t+l)}-1\right] \leqslant e^{r(t)}\left[\mathbf{E} e^{h e^{-r(t)} Z(t)}-1\right],
$$

where

$$
L(Y(l)):=\sup \left\{h \geqslant 0: \mathbf{E} e^{h Y(l)} \leqslant 1\right\} .
$$

Assume also that for any $t \geqslant 0$,

$$
e^{-r(t+l)} \Lambda^{\prime}(t+l) \leqslant e^{-r(t)} \Lambda^{\prime}(t) \quad \text { and } \quad e^{-r(t+l)} p^{\prime}(t+l) \geqslant e^{-r(t)} p^{\prime}(t) .
$$

Then under the assumptions of Theorem 2.3. all the assertions of that theorem hold for each $h \in[0, L(Y(l))]$ with

$$
\sup _{t \geqslant 0} \mathbf{E} e^{h Y(t)}=\sup _{0 \leqslant t \leqslant l} \mathbf{E} e^{h Y(t)} .
$$

In addition, for any $u>0$,

$$
\psi(u) \leqslant \inf _{h \in[0, L(Y(l))]}\left\{e^{-h u} \sup _{0 \leqslant t \leqslant l} \mathbf{E} e^{h Y(t)}\right\} \leqslant C_{2} e^{-L(Y(l)) u},
$$

where $C_{2}:=\sup _{0 \leqslant t \leqslant l} \mathbf{E} e^{L(Y(l)) Y(t)}$.

Proof. For any $t>l$, the random variables $\Delta_{t, l}:=Y(t)-Y(t-l)$ and $Y(t-l)$ are independent, so

$$
\mathbf{E} e^{h Y(t)}=\mathbf{E} e^{h Y(t-l)} \mathbf{E} e^{h \Delta_{t, l}}
$$

Let

$F(t):=\int_{t-l}^{t} G(x) d x$, where $G(x):=-h e^{-r(x)} p^{\prime}(x)+\left(\mathbf{E} e^{h e^{-r(x)} Z(x)}-1\right) \Lambda^{\prime}(x)$.

Then

$$
\mathbf{E} e^{h \Delta_{t, l}}=e^{F(t)} .
$$

Under the conditions (3.4) and (3.6) we have

$$
\begin{aligned}
F^{\prime}(t)= & G(t)-G(t-l) \\
= & h\left(e^{-r(t-l)} p^{\prime}(t-l)-e^{-r(t)} p^{\prime}(t)\right)+\left(\mathbf{E} e^{h e^{-r(t)} Z(t)}-1\right) \Lambda^{\prime}(t) \\
& -\left(\mathbf{E} e^{h e^{-r(t-l)} Z(t-l)}-1\right) \Lambda^{\prime}(t-l) \\
= & h\left(e^{-r(t-l)} p^{\prime}(t-l)-e^{-r(t)} p^{\prime}(t)\right)+e^{-r(t)} \Lambda^{\prime}(t) \cdot e^{r(t)}\left[\mathbf{E} e^{h e^{-r(t)} Z(t)}-1\right] \\
& -e^{-r(t-l)} \Lambda^{\prime}(t-l) \cdot e^{r(t-l)}\left[\mathbf{E} e^{h e^{-r(t-l)} Z(t-l)}-1\right] \\
\leqslant & 0 .
\end{aligned}
$$


Thus $F(t)$ is non-increasing and

$$
\mathbf{E} e^{h \Delta_{t, l}}=e^{F(t)} \leqslant e^{F(l)}=\mathbf{E} e^{h Y(l)} \leqslant 1, \quad \forall h \in[0, L(Y(l))] .
$$

Therefore,

$$
\mathbf{E} e^{h Y(t)} \leqslant \mathbf{E} e^{h Y(t-l)}, \quad \forall h \in[0, L(Y(l))] .
$$

Similar to the proof of Corollary 3.1 and 3.8 we can derive that for all $t>l$ and $h \in[0, L(Y(l))]$,

$$
\mathbf{E} e^{h Y(t)} \leqslant \sup _{0 \leqslant t \leqslant l} \mathbf{E} e^{h Y(t)} .
$$

Hence

$$
\sup _{0 \leqslant t \leqslant l} \mathbf{E} e^{h Y(t)} \leqslant \sup _{t \geqslant 0} \mathbf{E} e^{h Y(t)} \leqslant \sup _{0 \leqslant t \leqslant l} \mathbf{E} e^{h Y(t)}, \quad \forall h \in[0, L(Y(l))] .
$$

The desired results now follow from (3.9), Theorem 2.3 and Corollary 2.2.

The following corollary is a special case of Theorem 3.1 .

COROLLARY 3.2. Suppose there exists a real number $l>0$ such that for all $t \geqslant 0$ the claim sizes $Z(t+l)$ and $Z(t)$ are identically distributed. Moreover, assume that (3.6) holds. Then the assertions of Theorem 3.1 still hold.

A model satisfying the assumptions of Corollary 3.1 or Theorem 3.1 will be called quasi-periodic. A model satisfying the assumptions of Corollary 3.2 with

$\forall t \geqslant 0, \quad e^{-r(t+l)} \Lambda^{\prime}(t+l)=e^{-r(t)} \Lambda^{\prime}(t) \quad$ and $\quad e^{-r(t+l)} p^{\prime}(t+l)=e^{-r(t)} p^{\prime}(t)$ is called periodic (or even purely periodic).

REMARK 3.1. Let $r(t) \equiv 0$ in Corollary 3.1 or Theorem 3.1. Then it is not difficult to see that similar results also hold for periodic risk models without interest rates.

REMARK 3.2. From Theorem 3.1 we find several evident advantages of our results compared with the results of Asmussen and Rolski [3]. Firstly, in Theorem 3.1 if we let $r(t)=0$ and let (3.4) and (3.6) be equalities, then we can obtain the results of [3]. Secondly, from Remark 2.1 and (3.5) it is easy to see that our estimate is better. Thirdly, we do not use a relatively difficult change of measure which needs technical details. Our proof is based on a martingale approach with fewer assumptions.

EXAMPLE 3.1. Suppose $l=2$ and the claim sizes $Z(t)$ have exponential distribution $f(x)=e^{-x}, x>0$. We also assume that $p^{\prime}(t)=4 t$ and $\Lambda^{\prime}(t)=1$. Then the assumptions of Theorem 3.1 are satisfied with $r(t)=0$. 
We can calculate that for $h<1$,

$$
\mathbf{E} e^{h Z(t)}=\int_{0}^{\infty} e^{h x} e^{-x} d x=1 /(1-h) .
$$

Then for any $t>0$,

$$
\begin{aligned}
\mathbf{E} e^{h S(t)} & =\exp \left\{-h \int_{0}^{t} 4 x d x+\int_{0}^{t}(h /(1-h)) d x\right\} \\
& =\exp \left\{-2 h t^{2}+t h /(1-h)\right\} .
\end{aligned}
$$

It is easy to see that

$$
L(S(2))=\sup \left\{h \geqslant 0: \mathbf{E} e^{h S(2)} \leqslant 1\right\}=3 / 4 .
$$

Then by Theorem 3.1 , for any $u>0$ we have

$$
\psi(u) \leqslant \inf _{h \in[0,3 / 4]}\left\{e^{-h u} \sup _{0 \leqslant t \leqslant 2} \mathbf{E} e^{h S(t)}\right\} \leqslant(3 / 2) e^{-(3 / 4) u} .
$$

3.2. United risk model. Insurance companies can reduce the probability of ruin by investing their assets in risk-free assets or risky assets. In addition, some insurance companies may transfer part of the claims to the reinsurance company to reduce the probability of ruin. More details about investment and reinsurance can be found in Schmidli [23] and Bai and Guo [4].

Here we consider an interesting risk model in which a number of claim surplus processes are incorporated such that the $i$ th claim surplus process is added at time $t^{(i)}$, which means that at $t^{(i)}$ the insurance company will get another premium income with new premium rate and may need to pay new claims. Here we call these surplus processes the new branches which start at time $t^{(i)}$.

Let $S^{(0)}(t), S^{(1)}(t), S^{(2)}(t), \ldots$ be a sequence of independent claim surplus processes such that for any $t>0$,

$$
S^{(i)}(t)=\sum_{k=1}^{N^{(i)}(t)} Z^{(i)}\left(T_{k}^{(i)}\right)-p^{(i)} t, \quad i=0,1, \ldots, \quad \text { with } \quad S^{(i)}(0)=0
$$

where $N^{(i)}(t)$ is the Poisson process with intensity $\lambda^{(i)}, p^{(i)}$ is the non-random premium rate and $\left\{Z^{(i)}\left(T_{k}^{(i)}\right), k=0,1,2, \ldots\right\}$ are i.i.d. claim sizes which are independent of $N^{(i)}(t)$ where

$$
T_{k}^{(i)}:=\min \left\{t \geqslant 0: N^{(i)}(t) \geqslant k\right\}, \quad k=0,1, \ldots
$$

From (2.5) it follows that for each $i=0,1,2, \ldots$ and any $h \geqslant 0$,

$$
\mathbf{E} e^{h S^{(i)}(t)}=e^{t a^{(i)}(h)} \quad \text { with } \quad a^{(i)}(h)=\lambda^{(i)}\left(\mathbf{E} e^{h Z^{(i)}}-1\right)-h p^{(i)} .
$$


Let $0=t^{(0)}<t^{(1)}<\cdots$ be a sequence of non-random times. Define a new claim surplus process

$$
S(t):=\sum_{i=0}^{\infty} S^{(i)}\left(\left(t-t^{(i)}\right)^{+}\right) .
$$

We may interpret $S^{(i)}(t)$ as a process describing the business of the $i$ th branching company which begins at time $t^{(i)}$.

Thus we consider the surplus process

$$
R(t)=u-S(t), \quad \forall t \geqslant 0,
$$

with initial surplus $R(0)=u>0$ and $S(t)$ defined in (3.11). In this case, we say that the insurer's surplus $R(t)$ defined in (3.12) varies according to the united risk model.

We can see that this model can also be studied by applying model A.

For any $t>0$ and $h \geqslant 0$ write

$$
\begin{aligned}
& a(h, t)=\sum_{i=0}^{\infty}\left(t-t^{(i)}\right)^{+} a^{(i)}(h), \\
& a_{k}(h)=\sum_{i=1}^{k}\left(t^{(k)}-t^{(i)}\right) a^{(i)}(h), \quad a(h)=\sup _{k \geqslant 0} a_{k}(h),
\end{aligned}
$$

and set

$$
\bar{L}:=\sup \{h \geqslant 0: a(h) \leqslant 0\}, \quad L^{(i)}:=\sup \left\{h \geqslant 0: a^{(i)}(h) \leqslant 0\right\} .
$$

COROLlary 3.3. In the above setting, suppose $S(t)$ defined in (3.11) is a separable process with independent increments. Then the Lundberg inequality in (1.1) holds with $L:=\bar{L}$ where

$$
L^{(0)} \geqslant \bar{L} \geqslant \inf _{i \geqslant 0} L^{(i)}
$$

Proof. First, it is clear that 3.15 holds. By 3.10, 3.11) and the property of independence, we get

$$
\begin{aligned}
\mathbf{E} e^{h S(t)} & =\prod_{i=0}^{\infty} \mathbf{E} e^{h S^{(i)}\left(\left(t-t^{(i)}\right)^{+}\right)}=\prod_{i=0}^{\infty} e^{\left(t-t^{(i)}\right)^{+} a^{(i)}(h)} \\
& =e^{\sum_{i=0}^{\infty}\left(t-t^{(i)}\right)^{+} a^{(i)}(h)}=e^{a(h, t)} .
\end{aligned}
$$

By $(3.13)$ it is obvious that

$$
\sup _{t \geqslant 0} a(h, t)=\sup _{k \geqslant 0} a_{k}(h),
$$

since $a(h, \cdot)$ is a piecewise linear function.

The rest of the proof goes as for Theorem 2.2, so we omit it here.

Next we present an example such that $\bar{L}=L^{(0)}$. 
EXAMPLE 3.2. Suppose that $L^{(0)}>0$ and

$$
\begin{gathered}
a^{(i)}\left(L^{(0)}\right)>0, \quad i=1,3,5,7, \ldots, \\
a^{(i-1)}\left(L^{(0)}\right)+a^{(i)}\left(L^{(0)}\right) \leqslant 0, \quad i=1,3,5,7, \ldots .
\end{gathered}
$$

This may be interpreted as saying that the claim surplus processes with numbers $1,3,5,7, \ldots$ have negative income. But before opening a "bad" branch we open a "good" branch with property 3.16. In this case $\bar{L}=L^{(0)}$.

REMARK 3.3. If $i=0$ in the united risk model then it degenerates into a homogeneous compound Poisson risk model with

$$
\mathbf{E} e^{h S(t)}=\exp \left\{t\left[\lambda^{(0)}\left(\mathbf{E} e^{h Z^{(0)}}-1\right)-h p^{(0)}\right]\right\} .
$$

\section{COMPARISONS WITH THE EXISTING RESULTS}

In this section, we compare our results on the non-homogeneous renewal risk model from Zhou, Sakhanenko and Guo [26] with the results of Andrulyte et al. [1], Kievinaite and Šiaulys [16], and Kizinevič and Šiaulys [18].

Consider a class of risk processes $R(t)$ with the following properties:

(1) $R(t)$ may have positive jumps only at random or non-random times $T_{1}, T_{2}, \ldots$ such that

$$
\forall k=1,2, \ldots, \quad T_{k+1}>T_{k}>T_{0}:=0 \quad \text { and } \quad T_{n} \rightarrow \infty \text { a.s. }
$$

(2) $R(t)$ is monotone on each interval $\left[T_{k-1}, T_{k}\right), k=1,2, \ldots$, and $R(0)=$ $u>0$.

Model R: Assume the $k$ th claim $Z_{k}$ occurs at time $T_{k}$, i.e.,

$$
-Z_{k}:=R\left(T_{k}\right)-R\left(T_{k}-0\right) \leqslant 0, \quad \theta_{k}:=T_{k}-T_{k-1}>0, \quad k=1,2, \ldots
$$

Suppose that on each interval $\left[T_{k-1}, T_{k}\right)$ the premium rate is $p_{k}$, i.e.,

$$
\forall t \in\left[T_{k-1}, T_{k}\right), \quad R(t)-R\left(T_{k-1}\right)=p_{k}\left(t-T_{k-1}\right), \quad k=1,2, \ldots
$$

Assume also that the random vectors

$$
\left(p_{k}, Z_{k}, \theta_{k}\right), \quad k=1,2, \ldots,
$$

are mutually independent.

Then conditions (1) and (2) hold and the random variables

$$
Y_{k}=R\left(T_{k-1}\right)-R\left(T_{k}\right)=Z_{k}-p_{k} \theta_{k}=Z_{k}-X_{k}, \quad k=1,2, \ldots,
$$


are also mutually independent, where $X_{k}:=p_{k} \theta_{k}$ is the non-negative accumulated premium over a time period $\theta_{k}$. Model $\mathrm{R}$ is also called a non-homogeneous renewal risk model; it is different from model A introduced in Section 2.1. The ruin probability of model $\mathrm{R}$ is, for any $u>0$,

$$
\psi(u)=\mathbf{P}\left(\sup _{k \geqslant 1} S_{k}>u\right),
$$

where $S_{k}=Y_{1}+\cdots+Y_{k}$. Theorem 1 of [26] states that for any $u>0$ and $h \geqslant 0$,

$$
\psi(u) \leqslant e^{-h u} \sup _{n \geqslant 1} \mathbf{E} e^{h S_{n}} .
$$

We are going to estimate $\mathbf{E} e^{h S_{n}}$ in (4.2) to obtain a further explicit upper bound of the ruin probability of a non-homogeneous renewal risk model and compare our estimation with the results in Andrulytè et al. [1], Kievinaite and Šiaulys [16], and Kizinevič and Šiaulys [18].

The following simple assertion will be useful.

LEMMA 4.1. Let random variables $Y_{1}, Y_{2}, \ldots$ have finite expectations. Then for any $h \geqslant 0$ and $m \geqslant 1$,

$$
M_{m}(h):=\max _{1 \leqslant k \leqslant m} \mathbf{E} e^{h S_{k}} \leqslant e^{h C_{m}} \mathbf{E} e^{h S_{m}},
$$

where

$$
0 \leqslant C_{m}:=\max _{1 \leqslant k \leqslant m} \mathbf{E} S_{k}-\mathbf{E} S_{m}<\infty .
$$

Proof. For any $1 \leqslant k \leqslant m$ and $h \geqslant 0$, by Jensen's inequality in a probability space we have

$$
\begin{aligned}
\mathbf{E} e^{h S_{m}} & =\mathbf{E} e^{h S_{k}} \cdot \mathbf{E} e^{h\left(S_{m}-S_{k}\right)} \geqslant \mathbf{E} e^{h S_{k}} \cdot e^{h\left(\mathbf{E} S_{m}-\mathbf{E} S_{k}\right)} \\
& \geqslant \mathbf{E} e^{h S_{k}} \cdot e^{-h C_{m}} .
\end{aligned}
$$

From (4.5) we immediately have

$$
\mathbf{E} e^{h S_{k}} \leqslant e^{h C_{m}} \mathbf{E} e^{h S_{m}} .
$$

Taking the maximum over $1 \leqslant k \leqslant m$ yields the result.

The following inequality is evident and useful for us: for any $h \geqslant 0$ and $1 \leqslant$ $m \leqslant n$,

$$
M(h):=\sup _{n \geqslant 1} \mathbf{E} e^{h S_{n}} \leqslant M_{m}(h) \cdot\left(\sup _{n>m} \mathbf{E} e^{h\left(S_{n}-S_{m}\right)} \vee 1\right) .
$$


In fact, for any $h \geqslant 0$ and $1 \leqslant m \leqslant n$,

$$
\begin{aligned}
M(h) & =\sup _{n \geqslant 1} \mathbf{E} e^{h S_{n}}=\left(\sup _{n \leqslant m} \mathbf{E} e^{h S_{n}}\right) \vee\left(\sup _{n>m} \mathbf{E} e^{h S_{n}}\right) \\
& =M_{m}(h) \vee\left\{\sup _{n>m} \mathbf{E} e^{h\left(S_{n}-S_{m}\right)} \cdot e^{h S_{m}}\right\} \\
& =M_{m}(h) \vee\left\{\mathbf{E} e^{h S_{m}} \cdot \sup _{n>m} \mathbf{E} e^{h\left(S_{n}-S_{m}\right)}\right\} \\
& \leqslant M_{m}(h) \cdot\left\{1 \vee \sup _{n>m} \mathbf{E} e^{h\left(S_{n}-S_{m}\right)}\right\},
\end{aligned}
$$

since $\mathbf{E} e^{h S_{m}} \leqslant M_{m}(h)$.

The estimations of $M(h)$ and $M_{n}(h)$ are the central technical problems in applications of (4.2). Many ideas in this direction are known. The most famous ones are connected with the papers of Bennett [5] and Hoeffding [14]. In this section, we recall and use some ideas from [5] and [14]. First, by using important limits of mathematical analysis such as $\lim _{n \rightarrow \infty}(1+1 / n)^{n}=e$ we have

$$
\mathbf{E} e^{h S_{n}}=\prod_{k=1}^{n} \mathbf{E} e^{h Y_{k}} \leqslant\left(\frac{1}{n} \sum_{k=1}^{n} \mathbf{E} e^{h Y_{k}}\right)^{n} \leqslant \exp \left\{\sum_{k=1}^{n}\left(\mathbf{E} e^{h Y_{k}}-1\right)\right\} .
$$

Next, set $g(x)=e^{x}-1-x \geqslant 0$ and note that

$$
\mathbf{E} e^{h Y_{k}}=1+h \mathbf{E} Y_{k}+\mathbf{E} g\left(h Y_{k}\right) .
$$

From [5] and [14] we see that $g(x)$ has many useful properties. It is well known that for each $k=1,2, \ldots$,

$$
Y_{k}=Z_{k}-X_{k} \quad \text { with } X_{k}, Z_{k} \geqslant 0 .
$$

Then

$$
\begin{aligned}
\mathbf{E} g\left(h Y_{k}\right) & =\mathbf{E}\left[g\left(h Y_{k}\right): Y_{k} \geqslant 0\right]+\mathbf{E}\left[g\left(h Y_{k}\right): Y_{k}<0\right] \\
& =\mathbf{E}\left[g\left(h Y_{k}^{+}\right)\right]+\mathbf{E}\left[g\left(-h Y_{k}^{-}\right)\right] \leqslant \mathbf{E} g\left(h Z_{k}\right)+\mathbf{E} g\left(-h X_{k}\right) .
\end{aligned}
$$

It is easy to see that for any $h, C \geqslant 0$,

$$
\mathbf{E} g\left(-h X_{k}\right) \leqslant \frac{h^{2}}{2} \mathbf{E}\left[X_{k}^{2}: 0<X_{k} \leqslant C\right]+h \mathbf{E}\left[X_{k}: X_{k}>C\right] .
$$

But the most remarkable is the following property: if $H>0$ then by using the error term of the Taylor expansion of $e^{x}$ at $x=0$ such that $\delta(x)=e^{x}-1-x=$ $x^{2} \int_{0}^{1}(1-t) e^{t x} d t$ and the definition of $g(x)$ with $x=h Z_{k}$ we have

$$
\begin{aligned}
& \forall h \in(0, H], \quad h^{-2} \mathbf{E} g\left(h Z_{k}\right)=\mathbf{E} \int_{0}^{1} Z_{k}^{2}(1-t) e^{t h Z_{k}} d t \\
& \leqslant \mathbf{E} \int_{0}^{1} Z_{k}^{2}(1-t) e^{t H Z_{k}} d t=H^{-2} \mathbf{E} g\left(H Z_{k}\right) .
\end{aligned}
$$


For $H, C>0$ set

$$
\begin{aligned}
A_{n}(C) & :=\sum_{k=1}^{n} \mathbf{E}\left[X_{k}: X_{k}>C\right], \\
B_{n}(H, C) & :=\frac{1}{H^{2}} \sum_{k=1}^{n} \mathbf{E} g\left(H Z_{k}\right)+\frac{1}{2} \sum_{k=1}^{n} \mathbf{E}\left[X_{k}^{2}: X_{k} \leqslant C\right] .
\end{aligned}
$$

From the above statements we can see that

$$
\begin{aligned}
\mathbf{E} e^{h S_{n}} & \leqslant \exp \left\{\sum_{k=1}^{n}\left[h \mathbf{E} Y_{k}+\mathbf{E} g\left(h Y_{k}\right)\right]\right\} \\
& \leqslant \exp \left\{h \mathbf{E} S_{n}+h A_{n}(C)+h^{2} B_{n}(h, C)\right\} .
\end{aligned}
$$

Then from 4.9 we have the following assertion.

COROLLARY 4.1. Suppose that for some integer $m>0$ and real numbers $h, C>0$,

$$
A_{n}(C)+h B_{n}(h, C) \leqslant-\mathbf{E} S_{n}, \quad \forall n \geqslant m .
$$

Then

$$
M(h) \leqslant e^{h C_{m}},
$$

where $C_{m}$ is defined in (4.4). In particular, for any $u>0$,

$$
\psi(u) \leqslant e^{h\left(C_{m}-u\right)} .
$$

Proof. From 4.9) and 4.10), it is easy to see that

$$
\mathbf{E} e^{h S_{n}} \leqslant \exp \left\{h \mathbf{E} S_{n}+h\left(-\mathbf{E} S_{n}\right)\right\}=1, \quad \forall n \geqslant m .
$$

Then by 4.3, 4.6 and 4.13) we obtain

$$
\begin{aligned}
M(h) & \leqslant M_{m}(h)\left(\sup _{n>m} \mathbf{E} e^{h\left(S_{n}-S_{m}\right)} \vee 1\right) \leqslant e^{h C_{m}} \mathbf{E} e^{h S_{m}}\left(\sup _{n>m} \mathbf{E} e^{h\left(S_{n}-S_{m}\right)} \vee 1\right) \\
& =e^{h C_{m}}\left(\mathbf{E} e^{h S_{m}} \vee \sup _{n>m} \mathbf{E} e^{h S_{n}}\right)=e^{h C_{m}} \cdot \sup _{n \geqslant m} \mathbf{E} e^{h S_{n}} \leqslant e^{h C_{m}} .
\end{aligned}
$$

4.12 is directly obtained from 4.2 and 4.11.

If 4.10) does not hold, the following fact will be useful.

COROLlARY 4.2. Suppose that for some integer $m>0$,

$$
A_{n}(C) \leqslant-c^{*} \mathbf{E} S_{n} \quad \text { with } c^{*} \in(0,1), \quad \forall n \geqslant m,
$$

and

$$
B_{n}(H, C) \leqslant C^{*}\left(-\mathbf{E} S_{n}\right) \quad \text { with } 0<H, C^{*}<\infty, \quad \forall n \geqslant m .
$$

Then all assertions of Corollary 4.1 hold with

$$
0<h \leqslant \min \left\{H,\left(1-c^{*}\right) / C^{*}\right\} .
$$


Proof. It follows from (4.7) and (4.8) that $B_{n}(h, C) \leqslant B_{n}(H, C)$ for $h \in$ $(0, H]$. From this fact and 4.15$)$, for any $h \in(0, H]$ we have

$$
h B_{n}(h, C) \leqslant h B_{n}(H, C) \leqslant h C^{*}\left(-\mathbf{E} S_{n}\right) .
$$

Thus, inequality (4.17), with $h$ in 4.16), yields $h B_{n}(h, C) \leqslant\left(1-c^{*}\right)\left(-\mathbf{E} S_{n}\right)$, and hence (4.10) is satisfied in view of (4.14).

COROLlary 4.3. Suppose that

$$
\limsup _{n \rightarrow \infty} \mathbf{E} S_{n}<0, \quad \lim _{C \rightarrow \infty} \limsup _{n \rightarrow \infty} \frac{A_{n}(C)}{-\mathbf{E} S_{n}}<1,
$$

and that for some $H>0$ and any $C>0$,

$$
\limsup _{n \rightarrow \infty} \frac{B_{n}(H, C)}{-\mathbf{E} S_{n}}<\infty .
$$

Then there exists $h>0$ for which (4.11) and (4.12) hold.

Proof. It follows from (4.18) that there exists an integer $m>0$ such that $\sup _{n \geqslant m} \mathbf{E} S_{n}<0$ and 4.14) holds with some $c^{*} \in(0,1)$. Then we see from (4.19) that 4.15) is evidently true with some $C^{*}<\infty$. So, Corollary 4.3 follows from Corollary 4.2 .

REMARK 4.1. It is clear that

$$
\mathbf{E} g\left(H Z_{k}\right) \leqslant \mathbf{E} e^{H Z_{k}}-1 \leqslant \mathbf{E} e^{H Z_{k}}
$$

since $H>0$ and $Z_{k}$ is a non-negative random variable. And for all $C>0$,

$$
\mathbf{E}\left[X_{k}^{2}: X_{k} \leqslant C\right] \leqslant C^{2} \mathbf{P}\left[X_{k}>0\right] \leqslant C^{2} .
$$

It is not difficult to verify that from these facts and Corollaries 4.2 and 4.3 all assertions in [1], [16], [18] can be obtained.

Moreover, in this way we may obtain better constants than in those papers, since their authors use

$$
\mathbf{E} g\left(H Z_{k} / 2\right) \leqslant \frac{1}{2}\left(\frac{H}{2}\right)^{2} \mathbf{E}\left[Z_{k}^{2} e^{H Z_{k} / 2}\right] \leqslant 2 e^{-2} \mathbf{E} e^{H Z_{k}}
$$

instead of (4.7).

Note that we may always put $Z_{k}=Y_{k}^{+}$and $X_{k}=Y_{k}^{-}$. In this case (4.20) is not trivial with $\mathbf{P}\left[X_{k}>0\right]=\mathbf{P}\left[Y_{k}<0\right]$. 
EXAMPLE 4.1. Let independent random variables $Y_{1}, Y_{2}, \ldots$ have normal distributions such that

$$
\begin{aligned}
& B_{n}:=\operatorname{Var} S_{n} \rightarrow \infty, \\
& \forall k=1,2, \ldots, \quad \mathbf{E} Y_{k}:=-(27 / 64)\left(B_{k}+B_{k-1}\right) \operatorname{Var} Y_{k} .
\end{aligned}
$$

(We may, for simplicity, take $B_{k}:=k$.) In this case $\mathbf{E} S_{n}=-(27 / 64) B_{n}^{2}$ and

$$
\mathbf{E} e^{h S_{n}}=e^{h \mathbf{E} S_{n}+h^{2} \operatorname{var} S_{n} / 2}=e^{-(27 / 64) h B_{n}^{2}+h^{2} B_{n} / 2}=e^{f\left(B_{n}, h\right)} .
$$

It is easy to see that

$$
\sup _{n \geqslant 1} \mathbf{E} e^{h S_{n}}=\sup _{n \geqslant 1} e^{f\left(B_{n}, h\right)} \leqslant \sup _{x \geqslant 0} e^{f(x, h)}=e^{f(16 h / 27, h)}=e^{4 h^{3} / 27} .
$$

Hence

$$
e^{-h u} \sup _{n \geqslant 1} \mathbf{E} e^{h S_{n}} \leqslant e^{-h u+4 h^{3} / 27}=e^{g(h, u)} .
$$

Then for any $u>0$,

$$
\psi(u) \leqslant \inf _{h \geqslant 0} e^{-h u} \sup _{n \geqslant 1} \mathbf{E} e^{h S_{n}} \leqslant \inf _{h \geqslant 0} e^{g(h, u)}=e^{g((3 / 2) \sqrt{u}, u)}=e^{-u^{3 / 2}} .
$$

Thus, in the non-homogeneous case one can use $h=h(u) \rightarrow \infty$ as $u \rightarrow \infty$ to obtain a better bound than $\psi(u)=e^{-O(u)}$. This is impossible in the case of homogeneity.

Acknowledgments. The authors are grateful to the referees for their careful reading of the paper and helpful comments and suggestions.

\section{REFERENCES}

[1] I. M. Andrulytė, E. Bernackaite, D. Kievinaite and J. Šiaulys, A Lundberg-type inequality for an inhomogeneous renewal risk model, Modern Stoch. Theory Appl. 2 (2015), 173-184.

[2] S. Asmussen and H. Albrecher, Ruin Probabilities, World Sci., 2010.

[3] S. Asmussen and T. Rolski, Risk theory in a periodic environment: the Cramer-Lundberg approximation and Lundberg's inequality, Math. Oper. Res. 19 (1994), 410-433.

[4] L. Bai and J. Guo, Optimal proportional reinsurance and investment with multiple risky assets and no-shorting constraint, Insurance Math. Econom. 42 (2008), 968-975.

[5] G. Bennett, Probability inequalities for the sum of independent random variables, J. Amer. Statist. Assoc. 57 (1962), 33-45.

[6] E. Bernackaitė and J. Šiaulys, The finite-time ruin probability for an inhomogeneous renewal risk model, J. Industrial Management Optim. 13 (2017), 207-222.

[7] K. Blaževičius, E. Bieliauskienè and J. Šiaulys, Finite-time ruin probability in the inhomogeneous claim case, Lithuanian Math. J. 50 (2010), pp.260-270.

[8] A. Castañer, M. M. Claramunt, M. Gathy, C. Lefèvre and M. Mármol, Ruin problems for a discrete time risk model with non-homogeneous conditions, Scand. Actuar. J. 2013, 83-102.

[9] H. Cramér, On the mathematical theory of risk, in: Skandia Jubilee Vol. 2, Stockholm, 1930, $7-84$. 
[10] H. Cramér, Collective risk theory, Jubilee Volume, Skandia Insurance Company, 1955.

[11] D. C. M. Dickson, Insurance Risk and Ruin, Cambridge Univ. Press, 2005.

[12] H. Gerber, An Introduction to Mathematical Risk Theory, Univ. of Pennsylvania, 1979.

[13] J. Grandell, Aspects of Risk Theory, Springer, New York, 1991.

[14] W. Hoeffding, Probability inequalities for sums of bounded random variables, J. Amer. Statist. Assoc. 58 (1963), 13-30.

[15] Z. G. Ignatov and V. K. Kaishev, Two-sided bounds for the finite-time probability of ruin, Scand. Actuar. J. 2000, 46-62.

[16] D. Kievinaite and J. Šiaulys, Exponential bounds for the tail probability of the supremum of an inhomogeneous random walk, Modern Stoch. Theory Appl. 5 (2018), pp.129-143.

[17] J. F. C. Kingman, Poisson Processes, Clarendon Press, Oxford, 1993.

[18] E. Kizinevič and J. Šiaulys, The exponential estimate of the ultimate ruin probability for the non-homogeneous renewal risk model, Risks 6 (2018), 17 pp.

[19] C. Lefèvre and P. Picard, A nonhomogeneous risk model for insurance, Computers Math. Appl. 51 (2006), 325-334.

[20] F. Lundberg, Approximations of the probability function. Reinsurance of collective risks, Acad. Afhaddling, Almqvist och Wiksell, Uppsala, 1903 (in Swedish).

[21] J. Paulsen, Present value of some insurance portfolios, Scand. Actuar. J. 1997, 11-37.

[22] T. Rolski, H. Schmidli, V. Schmidt and J. L. Teugels, Stochastic Processes for Insurance and Finance, Wiley, Chichester, 1998.

[23] H. Schmidli, Stochastic Control in Insurance, Springer, London, 2007.

[24] A. Tuncel and F. Tank, Computational results on the compound binomial risk model with nonhomogeneous claim occurrences, J. Comput. Appl. Math. 263 (2014), 69-77.

[25] R. Vernic, On a conjecture related to the ruin probability for nonhomogeneous insurance claims, An. Ştiinţ. Univ. “Ovidius” Constanţa Ser. Mat. 23 (2015), 209-220.

[26] Q. Q. Zhou, A. Sakhanenko and J. Y. Guo, Lundberg-type inequalities for non-homogeneous risk models, Stoch. Models 36 (2020), 661-680.

Qianqian Zhou

School of Sciences

Nanjing University of Posts

and Telecommunications

Nanjing, 210023, P.R. China

E-mail: qianqzhou@yeah.net

Junyi Guo (corresponding author)

School of Mathematical Sciences

Nankai University

Tianjin, 300071, P.R. China

E-mail:jyguo@nankai.edu.cn
Alexander Sakhanenko

Sobolev Institute of Mathematics

4 Akad. Koptyug Avenue

Novosibirsk, 630090, Russia

and

School of Mathematical Sciences

Nankai University

Tianjin, 300071, P.R. China

E-mail: aisakh@mail.ru

Received 13.12.2019;

revised version 14.9.2020 
